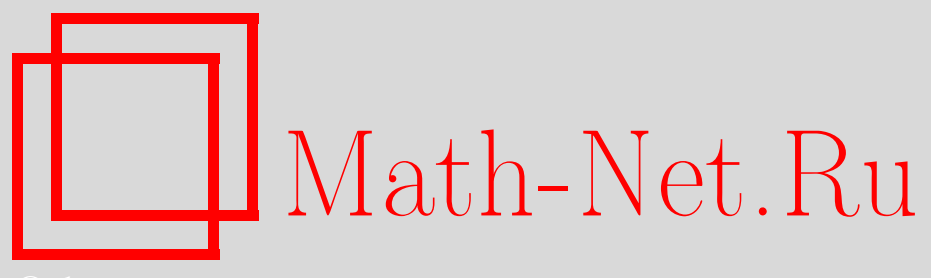

T. Shioda, Elliptic fibrations of maximal rank on a supersingular K3 surface, Изв. РАН. Сер. матем., 2013, том 77, выпуск 3, 139-148

DOI: https://doi.org/10.4213/im8017

Использование Общероссийского математического портала Math-Net.Ru подразумевает, что вы прочитали и согласны с пользовательским соглашением http://www . mathnet.ru/rus/agreement

Параметры загрузки:

IP: 18.209 .158 .208

26 апреля 2023 г., 13:51:43

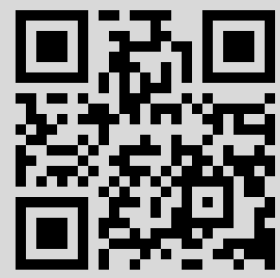


УДК 512.7

\title{
T. Shioda \\ Elliptic fibrations of maximal rank on a supersingular K3 surface
}

\begin{abstract}
We study a class of elliptic K3 surfaces defined by an explicit Weierstrass equation to find elliptic fibrations of maximal rank on K3 surface in positive characteristic. In particular, we show that the supersingular K3 surface of Artin invariant 1 (unique by Ogus) admits at least one elliptic fibration with maximal rank 20 in every characteristic $p>7, p \neq 13$, and further that the number, say $N(p)$, of such elliptic fibrations (up to isomorphisms), is unbounded as $p \rightarrow \infty$; in fact, we prove that $\lim _{p \rightarrow \infty} N(p) / p^{2} \geqslant(1 / 12)^{2}$.

Bibliography: 19 titles.
\end{abstract}

Keywords: K3 surface, Mordell-Weil lattice, Artin invariant.

DOI: $10.4213 / \operatorname{im} 8017$

To I.R. Shafarevich for his 90th birthday

\section{$\S 1$. Introduction}

For a smooth projective K3 surface $X$ defined over an algebraically closed field $k$, the Picard number $\rho:=\operatorname{rk} \mathrm{NS}(X)$ has a well-known upper bound:

$$
\rho \leqslant\left\{\begin{array}{lll}
h^{1,1}=20 & \text { if } \quad k=\mathbb{C}, \\
b_{2}=22 & \text { if } \quad p=\operatorname{char}(k)>0 .
\end{array}\right.
$$

When $X$ has an elliptic fibration with a section $\Phi: X \rightarrow \mathbf{P}^{1}$, that is, for an elliptic K3 surface $(X, \Phi)$, the Mordell-Weil rank $r$ has accordingly an upper bound:

$$
r \leqslant\left\{\begin{array}{lll}
18 & \text { if } \quad k=\mathbb{C} \\
20 & \text { if } \quad p=\operatorname{char}(k)>0 .
\end{array}\right.
$$

In this note, we focus on the case of maximal rank in (1.1) and (1.2).

First consider (1.1). The K3 surfaces over $k=\mathbb{C}$ with maximal Picard number $\rho=20$ are called singular K3 surfaces. The simplest examples are the Kummer surfaces of the product of two isogenous elliptic curves with complex multiplication, and they played an important role in the proof of the Torelli theorem for K3 surfaces in the celebrated work of Pyatetskii-Shapiro and Shafarevich [1]. A complete classification of singular K3 surfaces was given by Inose and Shioda [2], based on [1] and Kodaira's theory of elliptic surfaces [3], in terms of positive-definite even integral binary quadratic forms on the transcendental lattice of such a surface.

This work was partially supported by JSPS Grant-in-Aid for Scientific Research (C) 20540051. 
The K3 surfaces in positive characteristic with maximal $\rho=22$ are called supersingular K3 surfaces. By Artin [4], supersingular K3 surfaces form a 9-dimentional moduli space which has the stratification by means of the Artin invariant $\sigma$, $1 \leqslant \sigma \leqslant 10$, defined by $|\operatorname{det}(\mathrm{NS}(X))|=p^{2 \sigma}$. The simplest example of supersingular K3 surfaces are the Kummer surfaces of the product of two supersingular elliptic curves, which have $\sigma=1$. The uniqueness of the supersingular K3 surface with Artin invariant 1 is proved by Ogus [5].

Next, as to (1.2), we need to find a singular or supersingular K3 surface having an elliptic fibration without reducible fibres. The first example of complex elliptic K3 surfaces $(X, \Phi)$ with $r=18$ having an explicit defining equation was found by Kuwata [6] using Inose' fibration on Kummer surfaces [7], [8] and taking a suitable base change to kill the reducible fibres; more generally, he gets most values of the rank in the range $r \leqslant 18(k=\mathbb{C})$ in this way. In our previous papers [9]-[12], we studied the sections of these elliptic K3 surfaces using Mordell-Weil lattices [13].

In this note, we extend the above construction to characteristic $p>0$ to find K3 surfaces with an elliptic fibration of maximal rank 20. Thus we consider the elliptic K3 surfaces defined by the Weierstrass equation

$$
F_{\alpha, \beta}^{(n)}: y^{2}=x^{3}-3 \alpha x+\left(t^{n}+\frac{1}{t^{n}}-2 \beta\right),
$$

where $\alpha, \beta$ are arbitrary in $k$ and $n=1, \ldots, 6$. By Inose [8], $F_{\alpha, \beta}^{(2)}$ is isomorphic to the Kummer surface of the product of two elliptic curves $C_{1}, C_{2}$ :

$$
F_{\alpha, \beta}^{(2)} \cong\left(\operatorname{Km}\left(C_{1} \times C_{2}\right), \Phi\right),
$$

where $(\alpha, \beta)$ and the absolute invariants ${ }^{1} j_{1}, j_{2}$ of $C_{1}, C_{2}$ are related by

$$
\alpha^{3}=j_{1} j_{2}, \quad \beta^{2}=\left(1-j_{1}\right)\left(1-j_{2}\right)
$$

It will be shown among other things that the maximal rank $r=20$ is attained by taking $n=5$ or 6 and choosing $C_{1}, C_{2}$ to be non-isomorphic supersingular elliptic curves in the above construction.

Our main results are summarized in the next section. The idea behind the proof in characteristic $p$ will be discussed in $\S 3$, together with the necessary preparation, and the proof will be given in $\S 4$. Examples and remarks are given at the end of the note.

\section{§ 2. Main results for $p>0$}

Let $\operatorname{char}(k)=p>0$. In what follows we shall assume that

$$
p>3(\text { for } n \leqslant 4 \text { or } n=6) \text { and } p>5(\text { for } n=5) .
$$

\footnotetext{
${ }^{1}$ Throughout this paper, the absolute invariant $j$ is normalized in the classical way so that $j=1$ (instead of $j=12^{3}$ ) for $y^{2}=x^{3}-x$.
} 
THEOREM 2.1. For any $n \leqslant 6$, the rank of the Mordell-Weil lattice $\operatorname{MW}\left(F_{\alpha, \beta}^{(n)}\right)=$ $F_{\alpha, \beta}^{(n)}(k(t))$ is given by the formula

$$
r_{\alpha, \beta}^{(n)}=h+\operatorname{Min}\{4(n-1), 16\}-\left\{\begin{array}{lll}
0 & \text { if } \quad j_{1} \neq j_{2}, \\
n & \text { if } \quad j_{1}=j_{2} \neq 0,1, \\
2 n & \text { if } \quad j_{1}=j_{2}=0,1,
\end{array}\right.
$$

where $h$ denotes the rank of the group of homomorphisms $C_{1} \rightarrow C_{2}$ :

$$
h=\operatorname{rk} \operatorname{Hom}\left(C_{1}, C_{2}\right) \in\{0,1,2,4\} .
$$

As an immediate consequence, we have

Corollary 2.2. In characteristic $p>0$, we have

$$
r_{\alpha, \beta}^{(n)}=20 \Longleftrightarrow h=4, j_{1} \neq j_{2}, n=5,6 .
$$

Note that the case $h=\operatorname{rk} \operatorname{Hom}\left(C_{1}, C_{2}\right)=4$ occurs if and only if both $C_{1}$ and $C_{2}$ are supersingular elliptic curves in characteristic $p>0$. Let us denote by $\mathcal{H}_{p}$ the set of supersingular $j$-invariants in characteristic $p$ :

$\mathcal{H}_{p}:=\{j=j(C) \mid C$ is a supersingular elliptic curve in characteristic $p\}$.

We introduce some new notation:

$$
S_{j_{1}, j_{2}}^{(n)}:=F_{\alpha, \beta}^{(n)}, \quad M_{j_{1}, j_{2}}^{(n)}:=\operatorname{MW}\left(S_{j_{1}, j_{2}}^{(n)}\right),
$$

where

$$
\alpha=\sqrt[3]{j_{1} j_{2}}, \quad \beta=\sqrt{\left(1-j_{1}\right)\left(1-j_{2}\right)}
$$

(the choice of cube and square roots here is irrelevant).

TheOREm 2.3. Assume that $j_{1}, j_{2} \in \mathcal{H}_{p}$. Then, for any $n \leqslant 6$, the elliptic surface $S_{j_{1}, j_{2}}^{(n)}$ is a supersingular K3 surface of Artin invariant 1 , that is,

$$
\left|\operatorname{det}\left(\mathrm{NS}\left(S_{j_{1}, j_{2}}^{(n)}\right)\right)\right|=p^{2}
$$

If $j_{1} \neq j_{2}$ and $n=5$ or 6 , then the Mordell-Weil lattice $\operatorname{MW}\left(S_{j_{1}, j_{2}}^{(n)}\right)$ has maximal rank 20 and determinant $p^{2}$ :

$$
\operatorname{det} \operatorname{MW}\left(S_{j_{1}, j_{2}}^{(n)}\right)=p^{2} .
$$

Now recall that a supersingular K3 surface of Artin invariant 1 is unique up to isomorphism in a given characteristic $p$ by Ogus [5].

Corollary 2.4. For any $j_{1}, j_{2} \in \mathcal{H}_{p}$ and any $n \leqslant 6$, the $S_{j_{1}, j_{2}}^{(n)}$ are isomorphic to each other as K3 surfaces.

In the other direction, most of these K3 surfaces are not isomorphic as elliptic surfaces. In particular, this observation leads to the following assertion. 
TheOREM 2.5. Let $X(p)$ be a fixed supersingular K3 surface of Artin invariant 1 in characteristic $p$. Then for any $p>7, p \neq 13, X(p)$ admits at least two non-isomorphic elliptic fibrations of maximal rank 20. More precisely, if we denote by $N(p)$ the number of non-isomorphic elliptic fibrations of maximal rank 20 on $X(p)$, then we have

$$
N(p) \geqslant\left|\mathcal{H}_{p}\right| \cdot\left(\left|\mathcal{H}_{p}\right|-1\right) .
$$

Therefore $N(p)$ is unbounded as $p \rightarrow \infty$, and we have

$$
\lim _{p \rightarrow \infty} \frac{N(p)}{p^{2}} \geqslant\left(\frac{1}{12}\right)^{2} .
$$

\section{$\S$ 3. Preliminaries}

First we recall that the proof of the rank formula (2.2) in characteristic 0 is based on the following three facts.

1) The Picard numbers of two K3 surfaces $X, Y$ are equal if there is a dominant rational map $X \rightarrow Y$ (see [7] and [12, Lemma 3.1]).

2) The standard formula relating the Picard number of an elliptic surface $(S, \Phi)$ and the MW-rank is

$$
\rho=r+2+\sum_{v}\left(m_{v}-1\right)
$$

where the summation runs over $v \in \mathbf{P}^{1}$ such that $\Phi^{-1}(v)$ is a reducible fibre with $m_{v}>1$ irreducible components (see [13]).

3) The Picard number of a Kummer surface is

$$
\rho\left(\operatorname{Km}\left(C_{1} \times C_{2}\right)\right)=16+\rho\left(C_{1} \times C_{2}\right)=18+\operatorname{rk} \operatorname{Hom}\left(C_{1}, C_{2}\right) .
$$

In characteristic $p>0$, while 2) and 3) above continue to hold, the fact 1) is not always true. We have only the following weaker statement.

$1^{*}$ ) If there is a dominant map $X \rightarrow Y$ between two K3 surfaces, then

$$
\rho(X) \leqslant \rho(Y) .
$$

This follows from the inequality for the Lefschetz number $\lambda(X):=b_{2}(X)-\rho(X)$ :

$$
\lambda(X) \geqslant \lambda(Y)
$$

which holds more generally for any two surfaces with a dominant map $X \rightarrow Y$, and which implies, for example, that a unirational surface is supersingular in the sense that $\rho=b_{2}$ (cf. [14]).

Thus we proceed as follows. We shall construct suitable sublattices of the Mordell-Weil lattices

$$
M^{(n)}:=\operatorname{MW}\left(F_{\alpha, \beta}^{(n)}\right)
$$

to match the desired rank formula.

We refer to our previous paper [10] for proofs of the first two of the following lemmas. It is well-known that $\operatorname{Hom}\left(C_{1}, C_{2}\right)$ has the structure of an even integral 
lattice by taking the norm of a homomorphism $\varphi: C_{1} \rightarrow C_{2}$ to be $2 \operatorname{deg}(\varphi)$. (Note the change of notation: the norm of $\varphi$ was taken to be $\operatorname{deg}(\varphi)$ in [10].)

Given a lattice $L$, we denote by $L[n]$ the lattice structure on $L$ with the norm (or pairing) multiplied by $n$. For the root lattices $A_{2}, E_{8}$ and so on, we refer to [15].

Lemma 3.1 [10, Theorem 1.1]. Assume that $j_{1} \neq j_{2}$. Then there is a natural isomorphism

$$
M^{(1)} \simeq \operatorname{Hom}\left(C_{1}, C_{2}\right), \quad R_{\varphi} \leftrightarrow \varphi
$$

such that the height $\left\langle R_{\varphi}, R_{\varphi}\right\rangle$ of $R_{\varphi} \in M^{(1)}$ is equal to $2 \operatorname{deg}(\varphi)$.

Lemma $3.2\left[10\right.$, Theorem 1.2]. Assume that $j_{1} \neq j_{2}$. Then $M^{(2)}$ contains the sublattice

$$
M^{(2)} \supset M^{(1)}[2] \oplus\left(A_{2}^{*}\right)^{\oplus 2}[2]
$$

with finite index equal to $2^{h}$, where $A_{2}^{*}$ is the dual of the root lattice $A_{2}$. In particular, we have

$$
r^{(2)}=r^{(1)}+4, \quad r^{(1)}=h .
$$

Lemma 3.3. Let $n=2 m$ for $m=2$ or 3 . Assume that $j_{1} \neq j_{2}$. Then $M^{(n)}$ contains the sublattice

$$
M^{(n)} \supset M^{(m)}[2] \oplus E_{8}[2]
$$

with finite index equal to a 2-power. Thus we have

$$
r^{(4)}=r^{(2)}+8, \quad r^{(6)}=r^{(3)}+8 .
$$

Proof. This is based on the well-known fact that, given an elliptic curve $E / K$ and a separable quadratic extension field $K^{\prime} / K$, there is an elliptic curve $E^{\prime} / K$ (called the $K^{\prime} / K$-twist of $E / K$ ) such that we have an inclusion of Mordell-Weil groups,

$$
E\left(K^{\prime}\right) \supset E(K)+E^{\prime}(K) \supset 2 E\left(K^{\prime}\right)
$$

with $E(K) \cap E^{\prime}(K)$ a finite 2-torsion group. Applying this to the case $E=F_{\alpha, \beta}^{(m)}$, $m=2,3, K=k(t)$ and $K^{\prime}=k\left(t^{\prime}\right),\left(t^{\prime}\right)^{2}=2$, we find that

$$
F^{(n)}(k(t)) \simeq F^{(m)}\left(k\left(t^{\prime}\right)\right) \supset F^{(m)}(k(t))+E^{\prime}(K),
$$

where the twist

$$
E^{\prime}: y^{2}=x^{3}-3 \alpha t^{2} x+t^{3}\left(t^{m}+\frac{1}{t^{m}}-2 \beta\right)
$$

is a rational elliptic surface. It is easily seen that the Mordell-Weil lattice $E^{\prime}(K)$ is the root lattice $E_{8}$ (see [16]) since there are no reducible fibres under the assumption that $j_{1} \neq j_{2}$ and $m=2$ or 3 . Noting that the height of $P \in E(K)$ gets multplied by the degree $\left[K^{\prime}: K\right]=2$ as an element of $E\left(K^{\prime}\right)$ (see [13]), we have proved the assertion (3.9). (The same argument in the case $n=2, m=1$ proves Lemma 3.2.) 
Lemma 3.4. Assume that $n=5$ or 3 , and $j_{1} \neq j_{2}$. Then $M^{(n)}$ contains the sublattice

$$
M^{(n)} \supset M^{(1)}[n] \oplus \tilde{L}
$$

with finite index, where $\tilde{L}$ is a lattice of rank 16 for $n=5$ (or of rank 8 for $n=3$ ). Therefore we have

$$
r^{(3)}=r^{(1)}+8, \quad r^{(5)}=r^{(1)}+16 .
$$

Proof. We prove the case $n=5$ of the claim (3.14) here, and omit the case $n=3$ since the same argument works.

Denoting by $E / k(w)$ the elliptic curve:

$$
E: y^{2}=x^{3}-3 \alpha x+w-2 \beta,
$$

and letting $s=t+1 / t, T=t^{n}, w=T+1 / T$, we have

$$
M^{(n)}=E(k(t)), \quad M^{(1)}=E(k(T)) .
$$

Note (cf. [11]) that $k(t) / k(w)$ is a Galois extension with Galois group $G=\langle\sigma, \tau\rangle$, where $\sigma: t \rightarrow \zeta_{n} t, \tau: t \rightarrow 1 / t$ and $\zeta_{n}$ is a primitive $n$th root of unity. By Galois theory, the subfields $k(T), k(s)$ and $k(w)$ of $k(t)$ are respectively the fixed fields of $\langle\sigma\rangle,\langle\tau\rangle$ and $G$.

Now let $L=E(k(s))$ be the Mordell-Weil group of $k(s)$-rational points of

$$
E / k(s): y^{2}=x^{3}-3 \alpha x+\left(s^{5}-5 s^{3}+5 s\right)-2 \beta,
$$

which is a rational elliptic surface. The structure of the Mordell-Weil lattice on $L$ is again the root lattice $E_{8}$ under the assumption $j_{1} \neq j_{2}$. Next, let $L^{\prime}=L^{\sigma}=$ $E\left(k\left(s^{\prime}\right)\right)$ be the image of $L$ under $\sigma$, where $s^{\prime}=s^{\sigma}=\zeta t+1 /(\zeta t)$. Then $L^{\prime} \simeq E_{8}$ has rank 8 , and $\tilde{L}:=L+L^{\prime}$ (as a subgroup of $E(k(t))$ ) has rank 16 since $L \cap L^{\prime}=$ $E\left(k(s) \cap k\left(s^{\prime}\right)\right)=E(k(w))=\{0\}$. Thus we have the inclusion of lattices

$$
M^{(5)} \supset M^{(1)}[5]+\left(L+L^{\prime}\right)[2],
$$

where the two summands on the right-hand side are orthogonal to each other. (For more details and also for the fact that $\left(L+L^{\prime}\right)[2]=M_{\text {gen }}^{(5)}$, see [12, Lemmas 7.2-7.4].)

Hence the rank $r^{(5)}=\operatorname{rk} M^{(5)}$ satisfies

$$
r^{(5)} \geqslant r^{(1)}+16 .
$$

On the other hand, by (3.1) and the general inequality (3.3), applied to the rational $\operatorname{map} F^{(5)} \rightarrow F^{(1)}$, we have

$$
\rho^{(5)}=r^{(5)}+2 \leqslant \rho^{(1)}=r^{(1)}+2+8+8 .
$$

Thus we conclude that

$$
r^{(5)}=r^{(1)}+16
$$

which proves Lemma 3.4 in the case $n=5$ under consideration. 


\section{§4. Proofs of theorems}

4.1. Proof of Theorem 2.1. By collecting the results about the ranks $r^{(n)}$ obtained above, that is, by (3.8), (3.10) and (3.15), we have shown that, under the condition $j_{1} \neq j_{2}$,

$$
r^{(n)}=h+\operatorname{Min}\{4(n-1), 16\}, \quad h=\operatorname{rk} \operatorname{Hom}\left(C_{1}, C_{2}\right)
$$

for all $n \leqslant 6$, that is, we have the rank formula (2.2) in any characteristic $p$ as in $(2.1)$.

Thus we have proved Theorem 2.1 in the case $j_{1} \neq j_{2}$.

Next, to check the excluded case $j_{1}=j_{2}$, we can assume without loss of generality that $C_{1}=C_{2}=C$. Then the elliptic fibration on the Kummer surface $\operatorname{Km}\left(C_{1} \times C_{2}\right)$ under consideration, that is, the elliptic surface $F^{(2)}=F_{\alpha, \beta}^{(2)}$, has extra reducible fibres at $t \neq 0, \infty$, which arise from the graph of automorphisms of the elliptic curve $C$ (see [10, Proposition 7.1]). This decreases the rank by 2 , that is, to $r^{(2)}=$ $h+2$, if $\operatorname{Aut}(C)=\{ \pm 1\}$, and by 4 , that is, to $r^{(2)}=h$, if $|\operatorname{Aut}(C)|>2$. Then, considering the base change from $F^{(1)}$ to $F^{(2)}$, we easily see that in the case $n=1$, $r^{(1)}=h-1$ or $h-2=0$ according as $j(C) \neq 0,1$ or otherwise. Thus we have verified (2.2) for $n=1,2$ in case $j_{1}=j_{2}$.

The rest of the proof for $n=3, \ldots, 6$ and $j_{1}=j_{2}$ can be verified by modifying the arguments given in Lemmas 3.3 and 3.4 using the above result for $n=1,2$. This proves Theorem 2.1.

4.2. Proof of Theorem 2.3. Once the rank formula (2.2) is established, it is immediate that the Picard number of $S^{(n)}$ is independent of $n \leqslant 6$ :

$$
\rho\left(S_{j_{1}, j_{2}}^{(n)}\right)=\rho\left(F_{\alpha, \beta}^{(n)}\right)=h+18, \quad n \leqslant 6,
$$

for any $j_{1}, j_{2}$ and any $\alpha, \beta$, in view of (3.1).

We assume that $j_{1}, j_{2} \in \mathcal{H}_{p}, j_{1} \neq j_{2}$. Then we have $h=4$, and the elliptic surfaces $S_{j_{1}, j_{2}}^{(n)}$ are supersingular K3 for any $n \leqslant 6$. Let $\sigma(n)$ be the Artin invariant. We claim that

$$
\sigma(n)=1 \text { for all } n \leqslant 6 .
$$

This is known to be true for $n=2$ since $S^{(2)} \simeq \operatorname{Km}\left(C_{1} \times C_{2}\right)$ with supersingular elliptic curves $C_{1}, C_{2}$.

We consider the determinants of Modell-Weil lattices. Let

$$
d^{(n)}:=\operatorname{det} M^{(n)}=\operatorname{det} \operatorname{MW}\left(S_{j_{1}, j_{2}}^{(n)}\right), \quad 1 \leqslant n \leqslant 6 .
$$

For $n=1$, Lemma 3.1 implies that

$$
d^{(1)}=\operatorname{det} \operatorname{Hom}\left(C_{1} \times C_{2}\right)=\operatorname{det} \operatorname{NS}\left(C_{1} \times C_{2}\right)=p^{2} .
$$

For $n>2$, we shall see that $d^{(n)}$ is equal to $d^{(1)}$ times some rational number without a $p$-factor, using lemmas in the previous section.

First, for $n=2 m, m=2,3$, Lemma 3.3 implies that

$$
d^{(n)}=\operatorname{det}\left(M^{(m)}[2]\right) \operatorname{det}\left(E_{8}[2]\right) / \nu^{2}=d^{(m)} \cdot(2 \text {-power }),
$$


where $\nu$ is the index in (3.3) and a 2-power. Since $\operatorname{det} E_{8}=1$, both $d^{(4)} / d^{(2)}$ and $d^{(6)} / d^{(3)}$ are 2-powers. Similarly, Lemma 3.2 implies that $d^{(2)} / d^{(1)}$ is of the form $2^{a} 3^{b}$ since $\operatorname{det} A_{2}^{*}=1 / 3$.

Next, for $n=5$ or $n=3$, Lemma 3.4 implies that

$$
d^{(n)}=\operatorname{det}\left(M^{(1)}[n]\right) \operatorname{det}(\tilde{L}[2]) / \nu^{2},
$$

where $\nu$ is the index in (3.14). By [12], we have

$$
\operatorname{det} \tilde{L}=\operatorname{det} M_{\text {gen }}^{(n)}=\left\{\begin{array}{lll}
5^{4} & \text { if } & n=5, \\
3^{4} / 4^{2} & \text { if } & n=3 .
\end{array}\right.
$$

It follows that $d^{(3)} / d^{(1)}$ and $d^{(5)} / d^{(1)}$ is a rational number of the form $2^{a} 3^{b} 5^{c} / \nu^{2}$.

Therefore the $p$-factor of $d^{(n)}, n \neq 5$, is at most that of $d^{(1)}=p^{2}$ if $p>3$, and the $p$-factor of $d^{(5)}$ is likewise at most $p^{2}$ if $p>5$. We conclude that the $p$-factor of $d^{(n)}$ is at most $p^{2}$ for all $n \leqslant 6$, which implies the claim (4.3) that $\sigma(n)=1$ for all $n$.

Finally, for $n=5,6$ where the trivial lattice is unimodular, $d^{(n)}$ is equal to $\left|\operatorname{det} N S\left(S^{(n)}\right)\right|$, which implies that $\operatorname{det} M^{(n)}=p^{2}$.

4.3. Proof of Theorem 2.5. We first note that

LEMMA 4.1. The elliptic fibrations of the following surfaces ${ }^{2}$

$$
S_{j_{1}, j_{2}}^{(5)}, \quad S_{j_{1}, j_{2}}^{(6)}, \quad j_{1} \neq j_{2}, \quad j_{1}, j_{2} \in \mathcal{H}_{p},
$$

are mutually non-isomorphic.

Proof. If two elliptic fibrations over $\mathbf{P}^{1}$ are isomorphic to each other, the configuration type of the Kodaira fibres must be the same, and the set of places of singular fibres in $\mathbf{P}^{1}$ for one fibration must be transformed to that for the other by an automorphism of $\mathbf{P}^{1}$. It is easy to check that this cannot happen among the fibrations in (4.9) by using the fact that they are induced from $S_{j_{1}, j_{2}}^{(1)}$ by the base changes $t \rightarrow t^{n}$ for $n=5$ or 6 . We omit the computation.

We are now ready to prove Theorem 2.5. Each surface in the list (4.9) is a supersingular K3 surface of Artin invariant 1 by Theorem 2.3, and hence it is isomorphic to a chosen $X(p)$ (by Ogus). Then (4.9) can be regarded as a set of elliptic fibrations of maximal rank 20 on $X(p)$, which are non-isomorphic to each other by the above lemma. In this way, we have proved the inequality (2.10). The cardinality $\left|\mathcal{H}_{p}\right|$ of the supersingular $j$-invariants in characteristic $p$ is given by the famous Deuring-Eichler-Igusa formula (see [17])

$$
\left|\mathcal{H}_{p}\right|=\frac{p-1}{12}+\cdots
$$

In particular, we have $\left|\mathcal{H}_{p}\right| \geqslant 2$ if $p=11$ or $p>13$. This completes the proof of Theorem 2.5.

\footnotetext{
${ }^{2}$ The subscript $j_{1}, j_{2}$ in $S_{j_{1}, j_{2}}^{(n)}$ is meant to be an unordered pair.
} 
4.4. Examples and remarks. Consider the elliptic curves

$$
C_{1}: y^{2}=x^{3}-1, \quad C_{2}: y^{2}=x^{3}-x
$$

with $j$-invariants $j_{1}=0$ and $j_{2}=1$. It is well known that we have $0 \in \mathcal{H}_{p}$ if $p \equiv-1(\bmod 3)$, and $1 \in \mathcal{H}_{p}$ if $p \equiv-1(\bmod 4)$. Hence if $p \equiv-1(\bmod 12)$, then $0,1 \in \mathcal{H}_{p}$, and we can apply Theorem 2.3. Therefore the elliptic surfaces

$$
S^{(n)}(p): y^{2}=x^{3}+t^{n}+\frac{1}{t^{n}}, \quad n \leqslant 6,
$$

in characteristic $p$ are mutually isomorphic as K3 surfaces, and $S^{(5)}(p)$ and $S^{(6)}(p)$ give two non-isomorphic elliptic fibrations of rank 20 on the supersingular K3 surface with Artin invariant $\sigma=1$.

Let $p=11$ and $n=5,6$. The Mordell-Weil lattice $M$ of $S^{(n)}(p)$ is a positivedefinite even integral lattice of rank 20 and determinant $p^{2}$. The minimal norm (height) of $M$ is 4 and the number of minimal sections, that is, the kissing number, is 12540 . The centre density of the lattice $M$ is $\delta(M)=1 / 11$. See [18] for more details about this lattice.

REMARK 1. Recall [15] that the densest lattice known in dimension 20 has the centre density $\delta\left(\Lambda_{20}\right)=1 / 8$. Thus, if we knew that the number $N(p)$ (of elliptic fibrations of rank 20 in Theorem 2.5) is at least 1 for $p=7$, the argument used above would have produced a lattice of rank 20 and with centre density $\delta(M)=1 / p=1 / 7$, a new record! But, as $\left|\mathcal{H}_{p}\right|=1$ for $p=7$, we cannot find $j_{1} \neq j_{2}$ to apply Theorem 2.3.

REMARK 2. If characteristic $p=3$, the method of this paper again gives the surface $y^{2}=x^{3}+t^{n}+1 / t^{n}$ (for $n \neq 3,6$ ), which is not an elliptic, but a quasielliptic, K3 surface, and the Mordell-Weil group is finite.

REMARK 3. In this note, we have considered elliptic fibrations of maximal rank on a supersingular K3 surface only in the case in which Artin invariant $\sigma=1$. As to their existence in the case $\sigma>1$, see [19, Ex. 8].

\section{Bibliography}

1. I. I. Pyatetskii-Shapiro and I. R. Shafarevich, "A Torelli theorem for algebraic surfaces of type K3", Izv. Akad. Nauk SSSR Ser. Mat., 35:3 (1971), 530-572; English transl. Math. USSR-Izv., 5:3 (1971), 547-588.

2. T. Shioda and H. Inose, "On singular K3 surfaces", Complex analysis and algebraic geometry, Iwanami Shoten, Tokyo, 1977, 119-136.

3. K. Kodaira, "On compact analytic surfaces. II", Ann. of Math. (2), 77:3 (1963), 563-626; "On compact analytic surfaces. III", Ann. of Math. (2), 78:1 (1963), 1-40.

4. M. Artin, "Supersingular K3 surfaces", Ann. Sci. École Norm. Sup. (4), 7 (1974), 543-567.

5. A. Ogus, "Supersingular K3 crystals", Journées de Géométrie Algébrique de Rennes, vol. II (Rennes 1978), Astérisque, 64, Soc. Math. France, Paris, 1979, 3-86.

6. M. Kuwata, "Elliptic K3 surfaces with given Mordell-Weil rank", Comment. Math. Univ. St. Paul., 49:1 (2000), 91-100. 
7. H. Inose, "On certain Kummer surfaces which can be realized as non-singular quartic surfaces in $\mathbf{P}^{3 "}$, J. Fac. Sci. Univ. Tokyo Sect. IA Math., 23:3 (1976), 545-560.

8. H. Inose, "Defining equations of singular K3 surfaces and a notion of isogeny", Proceedings of the International Symposium on Algebraic Geometry (Kyoto 1977), Kinokuniya Book Store, Tokyo, 1977, 495-502.

9. T. Shioda, "A note on K3 surfaces and sphere packings", Proc. Japan Acad. Ser. A Math. Sci., 76:5 (2000), 68-72.

10. T. Shioda, "Correspondence of elliptic curves and Mordell-Weil lattices of certain elliptic K3's.", Algebraic cycles and motives, v.2, London Math. Soc. Lecture Note Ser., 344, Cambridge Univ. Press, Cambridge, 2007, 319-339.

11. T. Shioda, "The Mordell-Weil lattice of $y^{2}=x^{3}+t^{5}-1 / t^{5}-11$ ", Comment. Math. Univ. St. Pauli, 56:1 (2007), 45-70.

12. T. Shioda, "K3 surfaces and sphere packings", J. Math. Soc. Japan, 60:4 (2008), $1083-1105$.

13. T. Shioda, "On the Mordell-Weil lattices", Comment. Math. Univ. St. Paul., 39:2 (1990), 211-240.

14. T. Shioda, "An example of unirational surfaces in characteristic p", Math. Ann., 211:3 (1974), 233-236.

15. J.H. Conway and N. J. A. Sloane, Sphere packings, lattices and groups, Grundlehren Math. Wiss., 290, Springer-Verlag, New York, 1988.

16. K. Oguiso and T. Shioda, "The Mordell-Weil lattice of a rational elliptic surface", Comment. Math. Univ. St. Paul., 40:1 (1991), 83-99.

17. J. Igusa, "Class number of a definite quaternion with prime discriminant", Proc. Nat. Acad. Sci. U.S.A., 44 (1958), 312-314.

18. H. Ohashi, Integral sections of some elliptic surface via the binary Golay code, RIMS-Preprint, 2010, http://www.kurims.kyoto-u.ac.jp/ pioggia/docu/11.pdf.

19. T. Shioda, "Supersingular K3 surfaces with big Artin invariant", J. Reine Angew. Math., 381 (1987), 205-210.

Tetsuji Shioda

Department of Mathematics, Rikkyo University,

Tokyo, Japan

E-mail: shioda@rikkyo.ac.jp
Поступило в редакцию

26.06 .2012 